Revue d'histoire de l'Amérique française

REVUE D.HISTOIRE DE L'AMÉRIQUE FRANÇAISE

\title{
La bibliothèque scientifique de l'abbé Léon Provancher
}

\section{Raymond Duchesne}

Volume 34, numéro 4, mars 1981

URI : https://id.erudit.org/iderudit/303903ar

DOI : https://doi.org/10.7202/303903ar

Aller au sommaire du numéro

Éditeur(s)

Institut d'histoire de l'Amérique française

ISSN

0035-2357 (imprimé)

1492-1383 (numérique)

Découvrir la revue

Citer cet article

Duchesne, R. (1981). La bibliothèque scientifique de l'abbé Léon Provancher. Revue d'histoire de l'Amérique française, 34(4), 535-556.

https://doi.org/10.7202/303903ar d'utilisation que vous pouvez consulter en ligne.

https://apropos.erudit.org/fr/usagers/politique-dutilisation/ 


\title{
LA BIBLIOTHÈQUE SCIENTIFIQUE DE L'ABBÉ LÉON PROVANCHER
}

\author{
RAYMOND DUCHESNE \\ Institut d'histoire et de \\ sociopolitique des sciences \\ Université de Montréal
}

Les inventaires en cours des bibliothèques privées et publiques du Canada français ${ }^{1}$ risquent fort de confirmer l'impression selon laquelle les ouvrages scientifiques n'ont guère eu la faveur de nos ancêtres et qu'ils ne constituent qu'une portion médiocre des catalogues. Aussi l'existence d'une bibliothèque scientifique, principalement consacrée à l'histoire naturelle et dont la plupart des volumes nous sont parvenus ${ }^{2}$, prend-elle un intérêt particulier, non seulement pour l'histoire des sciences, mais également pour l'histoire des imprimés et l'histoire des idées au Canada français.

Il s'agit de la collection des ouvrages scientifiques, livres et périodiques, ayant appartenu à l'abbé Léon Provancher. Celui-ci, que l'on a appelé le «Linné canadien» ${ }^{3}$, est l'une des figures

1 Nous pensons, bien sûr, aux travaux du Groupe de recherche en histoire de l'imprimé au Québec dont les résultats ont commencé à paraître: Yvan Lamonde, Les Bibliothèques de collectivités à Montréal (Montréal, BNQ, 1979).

2 Dès 1893, le gouvernement provincial achetait de la succession de Provancher sa bibliothèque et ses collections d'histoire naturelle. Alors que les collections étaient déposées au Musée de l'Instruction publique, les livres étaient remis à Narcisse-Eutrope Dionne, bibliothécaire de la Législature, et aussitôt dispersés dans le Fonds général de la Bibliothèque du gouvernement. (Lettre de N.-E. Dionne à l'abbé Victor-Alphonse Huard, 1 juin 1893, Archives du Petit Séminaire de Chicoutimi (APSC): Fonds Huard, dossier 59, pièce 82). En 1976, on procéda à la reconstitution de la bibliothèque de Provancher en faisant rechercher parmi les ouvrages scientifiques de la Bibliothèque de l'Assemblée ceux qui portaient sa signature. C'est ainsi que l'on retrouva un peu plus de 300 titres, formant au total près de 500 volumes. Cette collection, baptisée «Fonds Provancher», est aujourd'hui conservée au Département de biologie de l'Université Laval, avec les collections entomologiques du naturaliste. Il est évident, vu la manière dont il a fallu procéder pour constituer le Fonds Provancher, que la plupart des livres du naturaliste qui ne traitaient pas de science, mais d'histoire, de religion, de philosophie ou de littérature, sont restés sur les rayons de la Bibliothèque de l'Assemblée. La collection des ouvrages scientifiques de Provancher dont il est question ici ne représente donc que la partie la mieux connue de sa bibliothèque. 189-197.

3 Cf. abbé Arthur Robitaille, «Le Linné canadien», Canada français, 3, 14 (1926):

RHAF, vol. 34, no 4, mars 1981 
majeures de l'histoire des sciences au Canada français. Ardent avocat de la diffusion des connaissances scientifiques dans son milieu, il s'est distingué par la fondation du Naturaliste canadien, une des plus anciennes revues scientifiques canadiennes, et par l'oeuvre considérable qu'il a laissée sur la géologie, les plantes et les animaux du Québec. Ses travaux sur les insectes constituent la partie la plus importante de sa contribution à l'histoire naturelle de l'Amérique du Nord et lui assurent une place parmi les grands entomologistes nord-américains du $\mathrm{XIX}^{\mathrm{e}}$ siècle $^{4}$.

\section{L'abbé Léon Provancher}

Léon Provancher est né le 10 mars 1820 dans la paroisse de Bécancour, comté de Nicolet ${ }^{5}$. Après des études classiques et théologiques au séminaire de Nicolet, de 1834 à 1844, il entre dans le ministère paroissial. D'abord vicaire, puis curé, il est envoyé successivement dans une paroisse de la Beauce, puis à l'Isle-Verte et, en 1854, à Saint-Joachim. C'est dans cette cure, alors qu'il a près de quarante ans, qu'il renoue avec un intérêt de sa jeunesse: l'histoire naturelle. Il s'initie d'abord à la botanique, grâce à quelques ouvrages et aux indications de l'abbé Ed. Richard, curé de SaintFerréol et ancien professeur au Collège de Sainte-Anne-de-laPocatière ${ }^{6}$. Dès 1858 , il publie un Traité élémentaire de botanique (Québec, 118 pages), ouvrage «à l'usage des maisons d'éducation» et pour lequel il a largement utilisé les travaux de botanistes américains, Alfonso Wood et Asa Gray notamment ${ }^{7}$. La préface du Traité annonce le prochain travail auquel il songe: la compilation d'une flore canadienne, ouvrage qui manque encore à cette époque aux naturalistes du Canada ${ }^{8}$.

4 Cf. E.O. Essig, A History of Entomology (New York, MacMillan, 1931) et G.P. Holland, "L'abbé Léon Provancher, 1820-1892», dans Pioneers of Canadian Science (G.F.G. Stanley, ed., Toronto, University of Toronto Press, 1966), 44-53.

5 Outre de nombreuses notices biographiques, signalons l'oeuvre de l'abbé V.-A. Huard, La Vie et l'oeuvre de l'abbé Provancher (Québec, Garneau, 1926); également la Bio-bibliographie de l'abbé Léon Provancher (École de Bibliothécaires de l'Université de Montréal, 1949), compilée par Jean-Bernard Léveillé.

6 Cf. Wilfrid Lebon, Histoire du Collège de Sainte-Anne-de-la-Pocatière, 18271927 (Québec, Charrier et Dugal, 1948-1949, 2 vol.).

L'auteur avait utilisé également un traité d'horticulture français célèbre, Le Bon jardinier, dont il possédait une édition datée de 1854 (Paris, Librairie agricole de la maison rustique). Provancher publiera, en 1884, une deuxième édition du Traité, "entièrement refondue et mise en rapport avec le programme du Baccalauréat de l'Université Laval».

8 Pour connaître l'état de la botanique nord-américaine à cette époque et le contexte dans lequel parut cette flore, voir Jacques Rousseau et Bernard Boivin, «La Contribution à la science de la Flore canadienne de Provancher», Naturaliste canadien, 6(95) 1968): 1499-1530. Voir également: A. Hunter Dupree, Asa Gray 1810-1888 (Cambridge, Mass., 1959). 
Pour ce travail, le curé de Saint-Joachim s'efforce d'obtenir la collaboration de tous ceux qui, avant lui, au Canada français, se sont intéressés à la botanique: le juge David Roy ${ }^{9}$, de la Malbaie; Mgr John Edward Horan, évêque de Kingston, autrefois professeur au Séminaire de Québec; l'abbé Jean-Baptiste Ferland, titulaire de la chaire d'histoire de l'Université Laval, qui a fait quelques herborisations sur les côtes du Labrador $^{10}$, le notaire Glackmeyer ${ }^{11}$, de Beauport. Il fait lui-même quelques voyages d'herborisation; un au lac Saint-Jean en 1861; un autre, l'année suivante, dans le Haut-Canada et sur la rive américaine du lac Ontario. Chaque fois, il est accompagné de l'abbé Louis-Ovide Brunet, professeur de botanique à l'Université Laval. Enfin, il s'en remet, un peu trop lourdement peut-être, aux travaux des botanistes américains.

À la fin de 1862, paraît la Flore canadienne, ou description de toutes les plantes des forêts, champs, jardins et eaux du Canada (Québec, Darveau, 1862, 2 vol.), ouvrage dont la page-titre est illustrée d'une forêt de sapins auxquels se mêlent quelques cactus et quelques palmiers. Bien que le Canada de Provancher soit celui d'avant la Confédération, le titre dépasse le contenu véritable de cette flore car les omissions sont nombreuses et d'importance ${ }^{12}$. Plus grave encore, l'auteur a péché contre les règles fondamentales de la nomenclature en donnant à des espèces déjà décrites des noms qu'il jugeait mieux appropriés. Enfin, le bon abbé a reproduit sans l'indiquer les gravures qui ornaient le Manual of the Botany of the Northern United States d'Asa Gray ${ }^{13}$ : ce dernier, à qui Provancher fait candidement parvenir deux exemplaires de la Flore canadienne, ne manque d'ailleurs pas de le lui reprocher publiquement ${ }^{14}$.

9 Lettre de Roy à Provancher, 30 avril 1860. APSC: Fonds Provancher.

10 Abbé L.O. Brunet, «Notes sur les plantes recueillies en 1858 par M. l'abbé Ferland sur les côtes du Labrador, baignées par les eaux du Saint-Laurent», La littérature canadienne de 1850 à 1860 (Québec, 1863, 2 vol.), I: 367-374.

11 Lettre de Glackmeyer à Provancher, février 1863. APSC: Fonds Provancher.

12 Rousseau et Boivin, op. cit., 1503. En fait, ces derniers ne font que confirmer le jugement de Marie-Victorin sur l'ouvrage de Provancher: «Sciences naturelles au Canada. L'étude des sciences naturelles, son développement chez les Canadiens-Français», Revue canadienne, 5 (20) (1917): 357.

13 Depuis 1848, le Manual de Gray a connu au moins huit éditions, la dernière en 1950. Rousseau et Boivin, op. cit., 1508: «Tout probablement, Provancher a fréquenté la première édition, puis soit la deuxième [1856] ou la troisième [1858].» Le Fonds Provancher ne compte qu'une édition de l'ouvrage, la quatrième, datant de 1859.

14 American Journal of Science (Silliman's Journal), (35) (1863): 445. 
Après le succès mitigé que lui vaut la Flore canadienne auprès des naturalistes et du grand public ${ }^{15}$, Provancher délaisse peu à peu la botanique. Ce refroidissement momentané de son intérêt pour l'histoire naturelle coïncide avec son déménagement de SaintJoachim à la cure de Portneuf. Dans cette nouvelle charge, il se dépense en entreprises diverses: il fonde une pépinière, publie un ouvrage d'horticulture et d'arboriculture qui connaittra un honnête succès ${ }^{16}$, il intéresse ses paroissiens et quelques financiers de Québec à la formation d'une petite compagnie de navigation ${ }^{17}$; et, enfin, il fonde le Naturaliste canadien. Cependant, une mésentente croissante entre ce curé si remuant et ses paroissiens amène l'archevêque de Québec à accepter sa démission de la cure de Portneuf. «Invalide du sanctuaire», selon sa propre expression ${ }^{18}$, Provancher est mis à la retraite à l'âge de quarante-neuf ans. Après avoir vécu quelques années à Québec, il s'établit, en 1872, dans une petite maison de Cap-Rouge. C'est là qu'il s'éteindra le 23 mars 1892.

Sans fortune familiale, ne recevant du diocèse qu'une maigre retraite, ét incapable, maigré tous ses efforts, de se placer dans l'enseignement ou la fonction publique, Provancher se voit contraint, à partir de 1869 , de ne compter que sur ses propres ressources. L'homme, heureusement, ne manque pas d'initiative. Il publie des manuels scolaires ${ }^{19}$ et des brochures d'horticulture ${ }^{20}$; il rédige, de mai 1875 à décembre 1876, la Gazette des familles canadiennes et acadiennes; il fonde, en 1888, la Semaine religieuse de Québec; il réédite plusieurs fois le Verger canadien; il organise des pèlerinages en Terre-Sainte ${ }^{21}$; etc... Résolu à tirer parti de son intérêt pour l'histoire naturelle, il obtient en faveur du Naturaliste canadien un octroi annuel du gouvernement provincial. Pendant quelques années, il collabore en qualité d' «assistant-rédacteur», au Journal

15 En 1898, l'édition n'est pas encore épuisée; Huard, op. cit., 87.

16 Le Verger canadien (Québec, Darveau, 1862) sera réédité, avec des modifications plus ou moins importantes, en 1864, en 1874, en 1881 et, enfin, en 1885.

17 Lettre de Glackmeyer à Provancher, 24 avril 1865. APSC: Fonds Provancher.

18 Petite Faune entomologique du Canada: Les Coléoptères (Québec, Darveau, 1877), v.

19 Outre les deux éditions du Traité élémentaire de botanique, Provancher a publié un Tableau chronologique et synoptique des principaux faits de l'histoire du Canada (Québec, Darveau, 1859) et une Histoire du Canada (Québec, Delisle, 1884).

20 Les oiseaux insectivores et les arbres d'ornement et forestiers (Québec, 1874).

21 Ce qui lui fournit l'occasion de faire lui-même le voyage à peu de frais. Il en rapporte de plus, un journal qu'il publie sous le titre, De Québec à Jérusalem (Québec, Darveau, 1884). 
d'agriculture que publie le Département d'Agriculture ${ }^{22}$. En 1877, il vend au gouvernement provincial des collections entomologiques qui forment la base du Musée de l'Instruction publique ${ }^{23}$. En 1889, c'est au tour du Collège de Lévis d'acheter pour son musée diverses collections de Provancher ${ }^{24}$.

La simple énumération des multiples entreprises de Provancher ne permet pas de voir comment le naturaliste a pu constituer la bibliothèque scientifique indispensable à la réalisation de son oeuvre majeure, les nombreux volumes de la Petite Faune entomologique du Canada ${ }^{25}$. La préparation d'un ouvrage de taxonomie de cette envergure, dans lequel l'auteur entend dresser la liste de toutes les espèces présentes sur le territoire du Canada et qui l'oblige à décrire plus d'un millier d'espèces nouvelles, rend nécessaire la consultation d'ouvrages spécialisés et de revues savantes. Peut-être a-t-il pu trouver à Québec certains des ouṿrages nécessaires, soit à la Bibliothèque de la Législature, soit à la Bibliothèque de l'Université Laval, où Mgr Thomas-Étienne Hamel et FrançoisXavier Bélanger, conservateur du Musée zoologique, s'occupent d'entomologie, soit encore à la Bibliothèque de la Literary and Historical Society of Quebec, qui accueille vers 1870 des entomologistes comme William Couper et George John Bowles ${ }^{26}$. Cela

22 L'«État des comptes publics», joint aux Documents de la Session 1878-1879, précise que Provancher a reçu $\$ 312.20$, au 30 juin 1878 , à titre de salaire comme assistantrédacteur. Il semble que Provancher ait occupé cette fonction de août 1877 (lettre d'Ernest Gagnon à Provancher, 17 août 1877. APSC: Fonds Provancher) à novembre 1883 (lettre d'Ernest Gagnon à Provancher, 7 novembre 1883. Id.).

23 Sur l'histoire des collections entomologiques de Provancher, voir J.R. Barron, «Provancher's Collections of Insects», Nat. can., 4 (102) (1975): 387-591.

24 Lettre de l'abbé P.-A. Bégin à Provancher, 24 août 1889. APSC: Fonds Provancher. Voir également Barron, op. cit., 406.

25 L'histoire des différents volumes de la Petite Faune est extrêmement complexe. Provancher publiait généralement ses descriptions et identifications d'insectes, à mesure qu'il traitait des différents ordres, dans les pages du Naturaliste canadien, pour ensuite les reprendre, avec des corrections et des additions, dans les volumes de la Petite Faune. Le plus simplement possible, la Petite Faune apparaît comme suit: vol. I Les Coléoptères (1877. 1879), Vol. II Les Orthoptères, les Névroptères et les Hyménoptères (1878-1889), Vol. III Les Hémiptères (1886). En 1929, l'abbé V.-A. Huard, se considérant le disciple de Provancher, publie le Vol. IV Les Lépidoptères, à partir des manuscrits laissés par le naturaliste de Cap-Rouge.

26 En janvier 1865, Couper, Bowles et quelques autres amateurs de la Vieille Capitale, dont l'abbé Brunet et le peintre Cornélius Krieghoff, organisent une société d'entomologie, la Quebec Branch of the Entomological Society of Canada. Voir le Canadian Naturalist and Geologist, (2) (1865): 57-60. Voir également Jean-Marie Perron, «Histoire des Sociétés d'entomologie au Québec», Annales de la Société entomologique de Québec, 1-2 (19) (1974) : 18-27. 
n'est pas sûr. Voulant montrer en quoi consiste la difficulté de déterminer l'espèce d'un coléoptère, une fois le genre connu grâce au catalogue de Melsheimer ${ }^{27}$, Provancher écrit:

Des 13 espèces mentionnées dans le Catalogue, 6 ont été décrites par Leconte, 2 par Say, 2 par Fabricius, 1 par Kirby, 1 par DeJean et 1 par Eschscholtz. Les espèces de Leconte ont été publiées dans les Annals of Lyceum of Natural History de New York de 1824 à 1852; celles de Say dans le Journal of the Academy of Natural Sciences of Philadelphia en publication depuis 1817; celles de Fabricius dans le Systema Eleutheratorum, 1701; celle de Kirby dans la Fauna Boreali-Americana, 1837; celle de DeJean dans le Spécies général des Coléoptères, 1825-1831; et celle d'Eschscholtz dans le Zoologischer Atlas, 1829; avez-vous tous ces ouvrages à votre disposition? Nous n'avons pu en trouver un seul dans aucune des bibliothèques de Québec, pas même dans celle de l'Université Laval avec ses 60,000 volumes. ${ }^{28}$

Quoi qu'il en soit, au moment où commencent à paraître dans les livraisons de 1872 du Naturaliste canadien les premières parties de la Petite Faune entomologique, Provancher a déjà quitté Québec pour Cap-Rouge et n'a donc plus aussi facilement accès aux bibliothèques de la ville. Il doit d'abord et avant tout compter sur les ressources de sa propre bibliothèque.

Pour la constituer, il a bien sûr recours d'abord à des achats. Outre ce que peuvent lui procurer les libraires canadiens, Provancher obtient les livres dont il a besoin de libraires européens et américains. Des maisons, comme la librairie Deyrolle \& Fils ${ }^{29}$, de Paris, avec laquelle il est en correspondance, sont à même de répondre à ses demandes. D'autres maisons, telles que le Comptoir de Conchyliologie, aussi de Paris, la Naturalists' Agency de Cassino, établie à Salem (Mass.), ou Foote, de Philadelphie, peuvent lui fournir non seulement des ouvrages spécialisés, mais également des spécimens d'histoire naturelle et des instruments scientifiques. De plus, Provancher voyage beaucoup: en 1869, il va à Salem pour assister au congrès de l'American Association for the Advancement of Science et pousse jusqu'à Boston afin de visiter le Museum

27 Catalogue of the Described Coleoptera of the United States (Washington, 1853).

28 Nat. can., 11 (7) (1875): 355.

29 Deyrolle est également éditeur, se spécialisant dans les ouvrages d'histoire naturelle. Le Fonds Provancher compte une demi-douzaine de titres de cet éditeur. 
of Comparative Zoology d'Agassiz; en 1871, il est à Washington, où il visite la Smithsonian Institution; en 1876, il visite Philadelphie à l'occasion de la Centennial International Exhibition et rencontre quelques-uns de ses correspondants de l'Academy of Natural Sciences de la ville ${ }^{30}$; en 1881 et en 1884 , il visite Londres et Paris; en 1890, il fait son dernier grand voyage, à Chicago. Par conséquent, les occasions ne lui manquent pas d'acheter les ouvrages spécialisés qui lui sont nécessaires ${ }^{31}$.

Sa correspondance avec de nombreux naturalistes d'Europe, des États-Unis et du Canada lui fournit également le moyen d'enrichir sa bibliothèque. Parmi les 182 auteurs des monographies et des catalogues du Fonds déposé à l'Université Laval, au moins 37, soit à peu près le cinquième, sont des correspondants de Provancher. Entre naturalistes, les dons de volumes et de tirés à part accompagnent souvent les lettres et les échanges de spécimens ${ }^{32}$. D'autres envois, moins désintéressés ceux-là, sont régulièrement adressés au rédacteur du Naturaliste canadien par des auteurs qui veulent un peu de publicité ${ }^{33}$.

Par ailleurs, il se trouve que certains musées au XIX $\mathrm{X}^{\mathrm{e}}$ siècle distribuent gratuitement, à tout le moins à des correspondants choisis, leurs publications scientifiques. C'est le cas, notamment, du Musée royal d'histoire naturelle de Belgique, dont le conservateur est un correspondant régulier de Provancher ${ }^{34}$, et de la Smithsonian de Washington. Le Fonds Provancher compte une vingtaine de titres publiés par des musées, dont douze par la Smithsonian. Dans la seconde moitié du XIX ${ }^{\mathrm{e}}$ siècle, le National Museum, institution de la Smithsonian s'occupant tout spécialement d'anthropologie et d'histoire naturelle, est un des musées d'Amérique du Nord les plus actifs dans le domaine de la systématique des espèces entomologiques. Les catalogues et synopsis qui y sont préparés sont des ouvrages indispensables pour les naturalistes qui, comme Provancher, s'occupent de taxonomie.

30 Principalement George H. Horn et Ezra T. Cresson.

31 De Québec à Jérusalem, 694.

32 Ainsi, par exemple, Cresson envoie à compter de 1875 les Transactions of the Entomological Society. Lettre de Cresson à Provancher, 8 mai 1875. APSC: Fonds Provancher.

33 C'est dans cette intention qu'un botaniste autrichien, le baron von Thümen, envoie le premier volume de sa Mycotheca Universalis. Lettre de Thümen à Provancher, 19 novembre 1875. APSC: Fonds Provancher.

34 Alfred Preudhomme de Borre, dont le Fonds Provancher compte quelques ouvrages d'entomologie. 
Enfin, le naturaliste de Cap-Rouge peut compter, pour enrichir sa bibliothèque, sur les échanges de ses propres publications avec celles des chercheurs et des institutions étrangères. Ses traités de systématique botanique et entomologique, peu appréciés du grand public ${ }^{35}$, sont cependant fort recherchés par les naturalistes d'Amérique du Nord et d'Europe, et des échanges sont souvent proposés. Le Naturaliste canadien représente également une bonne valeur d'échange, lorsque Provancher désire obtenir les publications régulières de sociétés savantes et des revues spécialisées. Un hyménoptérologiste français, Henri Miot, lui propose, en 1877, le Bulletin de la Société des sciences de Semur contre le Naturaliste ${ }^{36}$. Il reçoit déjà, depuis 1869 , des revues plus en rapport avec son intérêt pour les espèces nord-américaines: l'American Naturalist ${ }^{37}$ et l'American Entomologist ${ }^{38}$. Lorsque, d'octobre 1883 à juillet 1885 , le Naturaliste cesse de paraître, les échanges ne cessent pas complètement. Dès la renaissance de la revue, Provancher écrit:

Nous prierons (sic) les Revues scientifiques qui échangeaient avec nous, ou qui désireraient le faire à l'avenir, de vouloir bien nous adresser leurs publications, leur promettant sans délai la réciproque. (...)

De plus grands remerciements encore aux revues scientifiques suivantes qui nous ont semblablement continué leurs envois: l'American Naturalist, le Canadian Entomologist, l'Entomologists Monthly Magazine de Londres, la Revue horticole de Belgique, le Canadian Science Monthly de la Nouvelle-Écosse, le Bulletin of the Torrey Botanical Club de New York, et l'Entomologica Americana de Brooklin, N.Y. ${ }^{39}$

Cela explique comment il se fait que la bibliothèque de Provancher soit si bien pourvue de collections et de revues d'histoire naturelle. Ce sont là des publications particulièrement importantes pour le travail du taxonomiste, mais difficiles à obtenir et représentant une dépense fixe considérable.

35 Résumant sans doute le sentiment commun, l'abbé Laflamme, lui-même un «savant» professeur de l'Université Laval, écrit: «Je me suis souvent dit en parcourant l'ancien Naturaliste, que ces interminables descriptions d'espèces, nouvelles ou autres, n'avaient rien de bien alléchant en elles-mêmes pour la grande masse des lecteurs.» Lettre à Huard, 6 mars 1893. APSC: Fonds Huard, 61, 18.

36 Lettre de Miot à Provancher, 17 novembre 1877. APSC: Fonds Provancher.

37 Lettre de A.S. Packard à Provancher, 4 mars 1869. APSC: Fonds Provancher.

38 Lettre de C.V. Riley à Provancher, mars 1869. APSC: Fonds Provancher.

39 Nat. Can., 1 (15) (1885): 9-10. 


\section{Le Fonds Provancher: une analyse}

Ces divers procédés permettent donc à Provancher, tout isolé et (relativement) impécunieux qu'il soit, d'obtenir les ouvrages scientifiques dont il a besoin. L'analyse détaillée du Fonds déposé à l'Université Laval fait ressortir à la fois le caractère spécialisé et la diversité de ses sources. Elle permet également de mieux comprendre selon quels besoins Provancher a constitué sa bibliothèque et le rapport de celle-ci à l'oeuvre scientifique du naturaliste.

Ce qui ressort en premier lieu d'une répartition des livres et des revues du Fonds Provancher selon les disciplines (Tableaux A et B), c'est que l'ensemble de l'histoire naturelle s'y trouve représenté. À cela rien d'étonnant, puisque Provancher s'est intéressé, successivement ou simultanément, à presque tous les domaines de l'histoire naturelle: la botanique, la géologie, l'ornithologie, l'entomologie, la malacologie, etc... sans oublier la biographie des savants illustres et les récits de voyage des naturalistes ${ }^{40}$.

Le caractère encyclopédique de sa bibliothèque, du moins telle que nous le laisse imaginer le Fonds déposé à l'Université Laval, s'explique également par l'obligation qui était faite au rédacteur du Naturaliste canadien de posséder les diverses branches de l'histoire naturelle. La tâche que pouvait représenter la préparation des douze numéros annuels du Naturaliste, compte tenu de l'absence presque totale de collaborateurs et de la nécessité de varier les sujets pour ne pas lasser le public, n'aurait pu être accomplie sans puiser dans les ressources d'une bibliothèque assez vaste et diversifiée. Celle de Provancher était bien pourvue d'ouvrages généraux tels que les Oeuvres complètes de Buffon, éditées par Flourens (Paris, Garnier, 1853-1855, 12 vol.), les Suites à Buffon (Paris, Librairie de Roret, 1834-1876, 59 vol.), ouvrage monumental, qui couvrait l'ensemble de l'histoire naturelle et auquel avaient contribué des savants tels que Lacordaire, Cuvier, Dujardin, LePeletier de Saint-Fargeau, Milne-Edwards, etc..

Malgré la multiplicité des branches de l'histoire naturelle représentées, le Fonds Provancher laisse clairement apparaître l'intérêt tout particulier du naturaliste pour la botanique et l'entomo-

40 En 1890, dans Une excursion aux climats tropicaux (Québec, Langlais, 2), il écrit: «Vingt fois en lisant des voyages de naturalistes, tels que ceux de Darwin, de Humboldt, d'Agassiz et autres, j'avais en imagination savouré leurs jouissances...» 


\begin{tabular}{|c|c|c|c|c|c|c|c|c|}
\hline \multicolumn{9}{|c|}{$\begin{array}{c}\text { Tableau A } \\
\text { Le Fonds Provancher } \\
\text { Volumes, brochures, tirés à part, etc... }\end{array}$} \\
\hline & 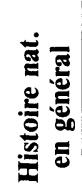 & 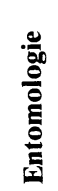 & 总 & 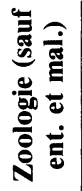 & $\frac{\stackrel{0}{0}}{\frac{0}{8}}$ & 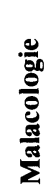 & 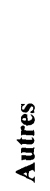 & हूّ \\
\hline États-Unis & 10 & 68 & 24 & 13 & 10 & 8 & 3 & 136 \\
\hline France & 3 & 25 & 11 & 14 & 4 & 5 & 2 & 64 \\
\hline Canada & 5 & 7 & 9 & 1 & 6 & 1 & 2 & 31 \\
\hline Angleterre & 2 & 10 & & & & 1 & 2 & 15 \\
\hline Belgique & & 6 & 1 & 1 & & 1 & & 9 \\
\hline Italie & & 6 & 1 & & & & & 7 \\
\hline Allemagne \& Autriche & 1 & 3 & 2 & & & 1 & & 7 \\
\hline Scandinavie & & 1 & & & & & & $\mathrm{i}$ \\
\hline Autres & 2 & & 1 & & & & 1 & 4 \\
\hline Total & 23 & 126 & 49 & 29 & 20 & 17 & 10 & 274 \\
\hline
\end{tabular}

Tableau B

\section{Le Fonds Provancher}

Revues et documents sériés.

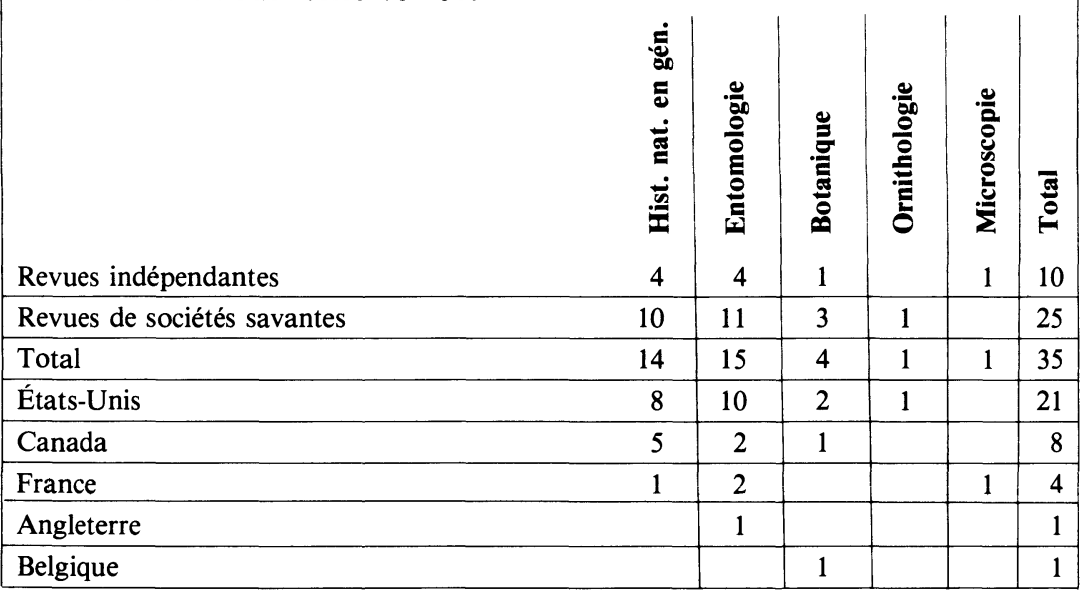


logie. Près des deux tiers des ouvrages formant le Fonds appartiennent à ces domaines de l'histoire naturelle. En outre, les ouvrages qui traitaient spécifiquement de la flore et des insectes nordaméricains surpassent largement en nombre ceux qui traitaient de points généraux, comme le Traité élémentaire d'entomologie (Paris, Baillière et Fils, 1873-1882, 3 vol.) de Maurice Girard ou Monograph of the Family of Nitidulariae (Londres, Taylor \& Francis, 1864) d'Andrew Murray; ou encore, de la flore et de la faune d'autres régions du globe, par exemple, Genera des coléoptères d'Europe (Paris, Deyrolle, 1857-1868, 4 vol.) de Jacquelin Du Val ou la Flore analytique des lichens de l'Orne (Autheuil, 1882-1884, 2 vol.) de l'abbé Henri Olivier.

Telle que nous la fait apparaître une première analyse du Fonds, la bibliothèque est celle d'un naturaliste s'intéressant à l'ensemble de l'histoire naturelle, mais plus spécialement à la botanique et à l'entomologie de l'Amérique du Nord. Cette constatation sur les spécialités de Provancher concorde parfaitement avec ce que nous savons de son oeuvre publiée. Cependant, il est possible de saisir plus finement encore les rapports entre la constitution de sa bibliothèque, les déplacements de son intérêt de la botanique à l'entomologie puis à la malacologie, et le progrès de son oeuvre scientifique de 1862 à sa mort.

En mettant en relation le sujet, la date et le lieu d'édition de 153 livres, tirés à part ou brochures du Fonds Provancher pour lesquels ces renseignements peuvent être obtenus (Tableau C), nous obtenons une vue de l'«âge» des différentes sections. Dans l'ensemble, les ouvrages publiés en Europe sont plus anciens que ceux publiés en Amérique du Nord (ou portant sur l'histoire naturelle de l'Amérique du Nord, puisque la coïncidence est parfaite). Près des deux tiers $(64 \%)$ des ouvrages européens formant le Fonds Provancher ont été publiés avant 1870 , contre $43 \%$ des ouvrages américains.

Une autre répartition fait apparaitre la même réalité. Parmi les 76 ouvrages parus avant 1869 (année médiane pour le groupe des 153 titres), près de $60 \%$ ont été publiés en Europe; pour les ouvrages publiés après, ce pourcentage tombe à $38 \%$.

Cela permet de distinguer dans le Fonds Provancher deux blocs d'ouvrages, de valeur à peu près égale quant au nombre. Le premier, plus ancien, est composé d'ouvrages publiés en Europe, portant le plus souvent sur l'ensemble de l'histoire naturelle, tels 


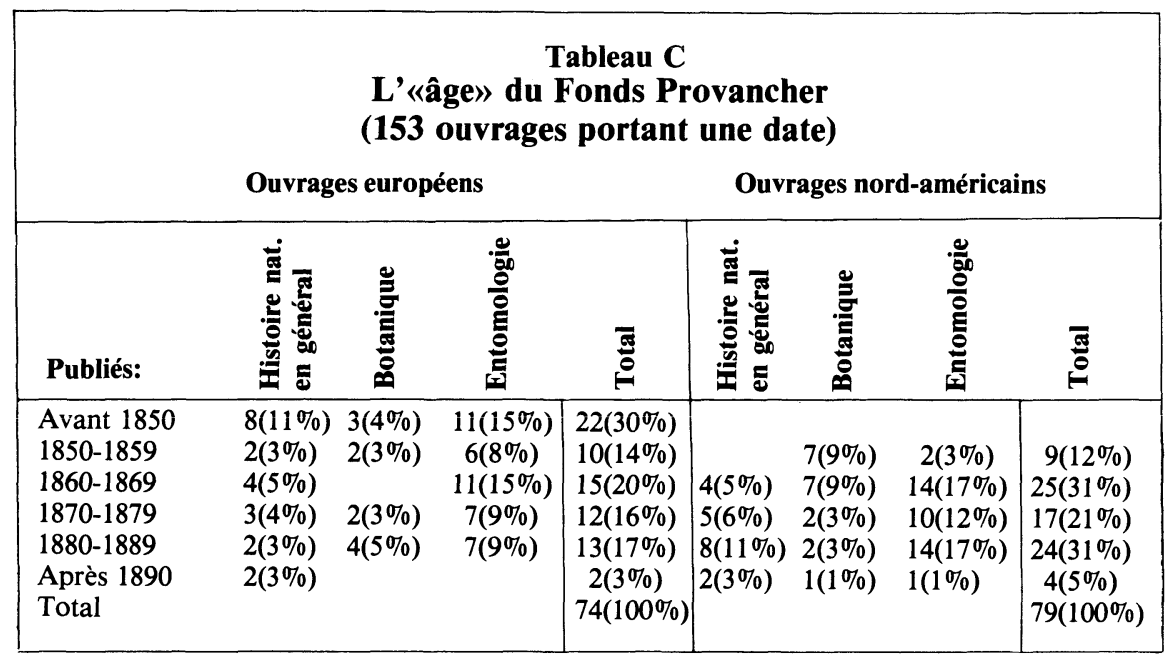

les dictionnaires et les ouvrages encyclopédiques déjà cités, ou sur la faune et la flore de diverses parties du monde à l'exception de l'Amérique du Nord. Le second bloc est formé d'ouvrages plus récents, publiés en Amérique et consacrés plus spécialement à l'entomologie nord-américaine. La formation de ces blocs serait principalement due aux changements d'intérêt de Provancher et à l'orientation de son travail vers la taxonomie des insectes nordaméricains à compter de 1870 .

Entre 1860 et 1870 , Provancher délaisse à peu près complètement la botanique pour l'entomologie. Aussi n'est-il pas surprenant de constater que le Fonds n'est guère pourvu d'ouvrages de botanique parus après cette dernière date. Dans ce domaine, les ouvrages les plus sérieux dont Provancher dispose sont le Manual of the Botany of the Northern United States (4e éd., New York, Ivison \& Phinney, 1859), A Class-Book of Botany (Boston, Crocker \& Brewster, 1856) d'Alfonso Wood et Botany of the Southern States (New York, Barnes \& Burr, 1860) de John Darby. Inversement, le nombre des titres consacrés à l'entomologie et publiés à compter de 1860 augmente sensiblement. Les ouvrages d'entomologie européens et nord-américains parus entre 1860 et 1869 se retrouvent à peu près en nombre égal: 11 contre 14 . Cependant, on observe parmi les ouvrages publiés après cette dernière date une nette diminution des ouvrages européens par rapport à ceux trai- 
tant de la faune entomologique d'Amérique du Nord: 14 contre 25. Cela s'explique sans doute par le fait qu'à mesure que Provancher se familiarise avec la taxonomie des espèces entomologiques nord-américaines et produit les volumes de la Petite Faune Entomologique, il doit s'appuyer davantage sur les catalogues américains et canadiens que sur les traités des auteurs européens.

Dès 1862, Provancher s'adresse à Carl Robert Osten Sacken, consul général de Russie à New York et entomologiste qui fait autorité sur les Diptères américains. Osten Sacken lui répond:

Vous désirez apprendre les publications principales sur l'entomologie américaine. Ce désir serait plus facile à satisfaire, si vous aviez indiqué une spécialité quelconque, un ordre de la nombreuse classe des insectes. ${ }^{41}$

Osten Sacken lui indique néanmoins, pour les différents ordres, les principaux auteurs américains; Leconte, Morris, Hagen, etc... de même que les ouvrages plus anciens de Say, Asa Fitch, Harris. À la même époque, Spencer F. Baird, de la Smithsonian, fait parvenir à Provancher quelques publications de son institution ${ }^{42}$. Il s'agit probablement des ouvrages de Hermann A. Hagen, Synopsis of the Neuroptera of North America (Washington, Smithsonian, 1861), de John Lawrence LeConte, Classification of the Coleoptera of North America (Washington, Smithsonian, 1861) et de John G. Morris, Synopsis of the Described Lepidoptera of North America (Washington, Smithsonian, 1862) ${ }^{43}$.

En 1863, Osten Sacken fait don à Provancher du premier volume de l'ouvrage important de Hermann Loew sur l'ordre des Diptères, Monograph of the Diptera of North America (Washington, Smithsonian, 1862-1873, 4 vol.) ${ }^{44}$.

À ces divers titres, il faut ajouter, parmi les ouvrages d'entomologie nord-américaine les plus importants du Fonds Provancher, des traités classiques comme ceux de Thomas Say ${ }^{45}$ et d'Alpheus S. Packard ${ }^{46}$, des monographies consacrées à divers ordres ou familles, comme celles de William H. Ashmead ${ }^{47}$ et de Ezra T.

41 Lettre de Osten Sacken à Provancher, 2 mai 1862. APSC: Fonds Provancher.

42 Lettre de Baird à Provancher, 26 avril 1862. APSC: Fonds Provancher.

43 Tous ces titres figurent au Fonds Provancher.

44 Lettre de Osten Sacken à Provancher, 17 février 1863. APSC: Fonds Provancher.

45 A Description of the Insects of North America (Ed. by J.L. LeConte, New York, Bouton, 1869).

46 Guide to the Study of Insects (Salem, Naturalists' Agency, 1869).

47 On the Cynipidous Galls of Florida (Jacksonville, 1886). 
Cresson ${ }^{48}$ sur les Hyménoptères, de John Bernhardt Smith ${ }^{49}$ sur les Lépidoptères, ou de Henri de Saussure ${ }^{50}$ sur les espèces américaines d'Hyménoptères solitaires. Ces ouvrages et les revues savantes, comme les Transactions of the American Entomological Society ou le Canadian Entomologist, sont les sources les plus utiles au travail du taxonomiste qui, comme Provancher, est davantage préoccupé de la détermination des spécimens que de l'arrangement des groupes supérieurs de la classification ou de l'étude des fonctions physiologiques.

Lorsque, dans les toutes dernières années de sa vie, le naturaliste de Cap-Rouge se tourne vers la malacologie et s'engage dans la publication d'un catalogue des mollusques de la province de Québec, il semble qu'il dispose déjà, dans ce nouveau domaine, des ouvrages indispensables. En 1891, il écrit:

Je possède un assez bon nombre d'auteurs sur les Mollusques, et ma collection de coquilles est assez considérable, je tâcherai de me tirer d'affaire du mieux que je pourrai. Je compte aux États-Unis des correspondants nombreux et complaisants, je recourrai à leur bienveillance lorsque je me trouverai dans l'embarras. ${ }^{51}$

De fait, figurent au Fonds Provancher des ouvrages de première importance: George W. Tryon, Structural and Systematic Conchology (Philadelphia, s.é., 1882-1884, 3 vol.) et Paul-Henri Fischer, Manuel de conchyliologie et de paléontologie conchyliologique (Paris, Savy, 1887, 2 vol.) ${ }^{52}$. Provancher reçoit également la revue américaine qui fait autorité dans ce domaine: le Nautilus, publiée à Philadelphie par l'American Association of Conchologists.

D'autres textes figurant au catalogue du Fonds Provancher méritent d'être signalés. En premier lieu, on relève une édition américaine, datant de 1864, du célèbre Journal of Research de Charles Darwin. D'abord publié en 1839, le livre consigne les observations que le jeune naturaliste a faites au cours du voyage

48 Synopsis of the Families and Genera of the Hymenoptera of America, North of Mexico (Philadelphia, Stockausen, 1887).

49 «A Revision of the Lepidopterous Family Saturniidae», U.S. Museum Proc. 9 (1887) : 414-437.

50 Synopsis of American Wasps: Solitary Wasps (Washington, Smithsonian, 1875).

51 Les Mollusques de la province de Québec (Québec, Darveau, 1891), iii.

52 Provancher recommande d'ailleurs ces deux ouvrages sur les mollusques aux lecteurs du Naturaliste: Nat. can., 11 (18) (1889) : 161-162. 
du H.M.S. Beagle autour du globe. Il fut réédité, en raison de son succès auprès du public, par Darwin lui-même, en 1845. Plus tard dans le siècle, après la publication de L'Origine des espèces, en 1859 , certains ont voulu y trouver les premiers éléments de la théorie de la sélection naturelle ${ }^{53}$. Il ne semble pas que Provancher ait été frappé de ces signes annonciateurs de la tempête, s'il est vrai qu'ils s'y trouvent, et qu'il ait vu dans le Journal autre chose qu'un agréable récit de voyage, où l'histoire naturelle figure en première place. En 1873, il en recommande la lecture à un de ses correspondants, Dominique-Napoléon Saint-Cyr, alors instituteur à SainteAnne-de-la-Pérade ${ }^{54}$. En 1887, il écrit:

D'abord croyant, Charles Darwin n'émit que timidement ses idées sur l'évolution des êtres. Nous avons lu très attentivement son voyage autour du monde (1832) et n'avons rien trouvé qui pût offenser la foi la plus sincère, nous y trouvons même le nom de Dieu plusieurs fois mentionné. ${ }^{55}$

Il faut signaler également quelques travaux de géologie; en particulier, ceux d'Henri $\mathrm{Ami}^{56}$ et de Jules Marcou ${ }^{57}$ consacrés aux formations de l'Est du Canada. Marcou, une des grandes figures de la géologie américaine et un des principaux protagonistes de la controverse sur le système taconique, avait plusieurs correspondants au Canada français ${ }^{58}$. Outre Ami, qui appartenait à la Commission géologique du Canada, on compte parmi eux Pierre-J.-Olivier Chauveau, Saint-Cyr et l'abbé J.-C.-K. Laflamme, titulaire de la chaire de géologie et de minéralogie de l'Université Laval. Les ouvrages d'Ami et de Marcou figurant au Fonds Provancher se rapportent, en partie tout au moins, au problème du groupe de Québec, formation géologique difficile à classer dans le contexte du débat sur le système taconique. On peut supposer que Marcou a adressé ses publications à Provancher, comme il l'avait fait pour

53 Voir, par exemple, l'opinion de Francis Darwin, le fils de Charles, sur cette question; The Autobiography of Charles Darwin (New York, Appleton, 1892), 179. L'histoire récente du darwinisme, cependant, n'a pas retenu le Journal en tant que première expression de la théorie; voir C. Limoges, La Sélection naturelle (Paris, P.U.F., 1970).

54 Lettre de Saint-Cyr à Provancher, 21 janvier 1873. APSC: Fonds Provancher.

55 «Le Darwinisme», Nat. can., 12 (16) (1887): 191. 61-66.

56 "The Utica Slate Formation", Ottawa Field-Naturalists' Club, Trans., 3 (1882):

57 «Notice sur les gisements des lentilles trilobitifères de la Pointe Lévis», Bulletin de la Soc. géologique de France, 21 (1864): 236-250.

58 Lettres de Marcou à l'abbé J.C.K. Laflamme aux Archives du Séminaire de Québec; notamment, 23 avril 1885 (Université 62, 58), 24 mai 1890 (Université 62, 64) et 13 juin 1891 (Université 62, 67). 
Laflamme, afin de se gagner un allié et dans l'espoir de voir le rédacteur du Naturaliste se jeter dans la mêlée.

Enfin, signalons quelques volumes de théologie naturelle, oeuvres de William Dawson ${ }^{59}$, paléobotaniste et Principal de McGill, et de Gerald Molloy ${ }^{60}$, vulgarisateur britannique. La controverse engendrée par Darwin avait amené certains partisans de la fixité des espèces à rénover quelque peu le vieil édifice de la théologie naturelle. Un des principaux problèmes des «creationists» consistait à établir la parfaite concordance du récit biblique de l'origine du monde et d'une vision fixiste des espèces, de manière à asseoir plus solidement cette dernière. Parmi les nombreux auteurs à avoir exploité ce filon, Dawson s'était distingué autant auprès des hommes de science que du grand public ${ }^{61}$.

Il n'est pas extraordinaire de voir apparaître ces quelques ouvrages de Darwin, de Marcou et de Dawson au catalogue du Fonds Provancher. En fait, il faut plutôt s'étonner de ne pas en trouver plus de cette sorte. La bibliothèque de Provancher étaitelle vraiment si peu fournie de "classiques» du débat transformiste, d'ouvrages de géologie, de théologie naturelle et d'apologétique? Cette question nous amène à nous tourner vers la correspondance et l'oeuvre publiée de Provancher, afin de voir dans quelle mesure ses sources bibliographiques pourraient avoir dépassé celles du Fonds déposé à l'Université Laval.

\section{La bibliothèque selon la correspondance et l'oeuvre}

La bibliothèque de Provancher dépassait très certainement le contenu du Fonds et un inventaire des oeuvres citées dans sa correspondance et ses propres ouvrages permet d'en compléter le catalogue. Une telle étude n'a pas simplement pour but d'établir quels ouvrages manquent au fonds de l'Université Laval, ce qui ne présente que peu d'intérêt. L'étude doit plutôt nous servir à mieux définir l'étendue de la bibliothèque dont le naturaliste disposait et, surtout, à vérifier si, dans les domaines de la théorie transformiste, de la géologie et de la théologie naturelle, cette bibliothèque était vraiment aussi pauvre que le catalogue du Fonds le laisse croire.

59 Modern Ideas of Derivation, Address as President of the Natural History Society of Montreal, (n.é., 1869, 18 pages).

60 Geology and Revelation (New York, Putnam, 1870). Il s'agit de l'édition américaine de l'ouvrage publié à Londres la même année.

61 Voir C.E. O'Brien, Sir William Dawson. A Life in Science and Religion (Philadelphia, American Philosophical Society, 1971). 
Outre les titres du Fonds de Laval, on trouve cités dans la correspondance et l'oeuvre publiée de Provancher plus d'une centaine d'autres que le naturaliste reconnaît explicitement avoir utilisés ou dont il a rendu compte dans les pages du Naturaliste canadien. Il s'agit de monographies, de documents officiels et de revues qui se rapportent non seulement aux différentes branches de l'histoire naturelle, mais aussi à l'histoire, à la grammaire, à la religion ou à la littérature. Parmi cette centaine de titres, les ouvrages canadiens figurent en bonne place ${ }^{62}$. On peut citer, particulièrement, le traité de l'abbé Brunet, Éléments de botanique et de physiologie végétale (Québec, Delisle, 1870), et celui de l'abbé Jean Moyen, professeur au Collège de Montréal, Cours élémentaire de botanique et Flore $d u$ Canada (Montréal, Desbarats, 1871), ouvrages dont Provancher a fait le compte rendu dans le Naturaliste canadien ${ }^{63}$. On peut penser également que Provancher avait dans sa bibliothèque les ouvrages de l'abbé Laflamme: Éléments de géologie et de minéralogie (Québec, Delisle, 1881) et Esquisse biographique de Michel Sarrazin (Québec, 1887), publications notées par le Naturaliste ${ }^{64}$. Enfin, Provancher connaissait sûrement le grand ouvrage de John Macoun, Catalogue of Canadian Plants (Ottawa, Geological Survey, 1883-1902, 7 vol.), qu'il attaque violemment, dans le Naturaliste, pour avoir ignoré les botanistes canadiens-français ${ }^{65}$.

$\mathrm{Au}$ nombre des revues savantes qu'il faut ajouter à celles du Fonds pour avoir une plus juste idée des sources de Provancher, on relève les Actes de la Société linnéenne de Bordeaux, qu'il recevait du Professeur Jean Pérez, de la Faculté des Sciences de l'Université de Bordeaux et officier de la Société66, les Zoological Record, qu'il échangeait contre le Naturaliste ${ }^{67}$, et les Transactions of the Ottawa Field-Naturalists' $\mathrm{Club}^{68}$. On peut citer aussi: Valley Naturalist, Archives of Science, Annales de la Société entomologique de Belgique, American Journal of Microscopy, etc...

62 Leur nombre s'élève à 37.

63 Il s'agit plutôt d'éreintements en règle que Provancher sert à l'ouvrage de Brunet, Nat. can., 5 (2) (1870): 144-150, ainsi qu'à celui de Moyen, Ibid., 13 (3) (1871): 379, 7 (4) (1872) : 229-232.

64 Nat. can., 9 (12) (1881): $269-270$ et 6 (17) (1887): 100.

65 Nat. can., 7 (15) (1886): 176-177.

66 Lettre de Pérez à Provancher, 5 janvier 1880. APSC: Fonds Provancher. En outre, une collection des Actes se trouve à la Bibliothèque de l'Assemblée.

67 Lettre de E.C. Rye, secrétaire de la Royal Geographical Society de Londres, à Provancher, 3 novembre 1875. APSC: Fonds Provancher.

68 Nat. can., 12 (13) (1882). 
Pour débattre du transformisme, Provancher s'est appuyé sur des sources qui ne figurent pas au Fonds, mais qui sont citées à quelques reprises dans le Naturaliste, notamment dans une longue suite d'articles consacrés au "Darwinisme» en $1887^{69}$. Mis à part l'ouvrage de Darwin, De l'origine des espèces ou lois du progrès chez les êtres organisés (traduit par Clémence-Auguste Royer, Paris, 1862), et un article récent de l'American Naturalist ${ }^{70}$, toutes les autres sources citées par Provancher - et il y en a peu - sont des textes de vulgarisation ou des articles de dictionnaire. Il s'agit, par exemple, des "Causeries sur le transformisme» prononcées par Henri Gadeau de Kerville devant la Société d'étude des sciences d'Elbeuf ${ }^{71}$, de l'article «Nature», signé par Cuvier dans le Dictionnaire des Sciences naturelles de Levreault, et d'un autre article «Instinct», signé par Flourens dans le Dictionnaire universel d'histoire naturelle d'Orbigny. Si Provancher n'éprouve pas le besoin de se référer à des travaux plus savants ou plus récents, c'est qu'il a choisi de placer sa discussion du transformisme non pas dans le cadre du débat scientifique, mais dans celui de la controverse religieuse. Dès le premier article de la série, identifiant le darwinisme à une hérésie au service des politiciens matérialistes français, «les Bert, les Hugo, les Ferry, les Goblet, les Clémenceau», Provancher répond à ceux qui voudraient distinguer la science et la doctrine révélée que «le transformisme est inséparable de la religion, puisqu'il sape la base de toute religiosité quelconque ${ }^{72}$. La suite ne consiste qu'à rappeler en quoi les propositions les plus générales de l'hypothèse transformiste représentent une contradiction possible de la Révélation et du dogme et doivent, pour cela, être condamnées.

L'examen de la correspondance de Provancher et des quelques travaux qu'il a consacrés à la géologie ne permet pas, non plus, d'ajouter de nombreux titres à ceux du Fonds traitant de cette science. Ainsi qu'il en a été pour le transformisme, Provancher aborde la géologie comme un terrain où il faut défendre le dogme menacé. Au début d'une série d'articles sur la géologie, il déclare:

69 «Le Darwinisme», Nat. can., 7, 8, 9, 12 (16) (1887); 3, 4, 6, 7, 9, (17) (1887).

70 Lester F. Ward, «Evolution in the Vegetable Kingdom», Am. Nat., 7 (19) (1885): $637-644$

71 Causeries sur le transformisme (Paris, Reinwald, 1887).

72 Nat. can., 7 (16) (1887): 108. 
...partout où elle est entendue, [la science] n'est en désaccord nulle part avec la Révélation; tout au contraire, les arguments qu'on avait cru trouver contre les vérités révélées, dans des découvertes et des observations imparfaites, sont venues, après de nouvelles études, à confirmer précisement le récit des livres saints ou du moins à demeurer en tous points d'accord avec lui. C'est cet accord de la Géologie avec la Révélation que nous nous efforcerons de mettre en relief dans les entretiens que nous exposerons sur ce sujet. ${ }^{73}$

Cet objectif posé, Provancher peut asseoir son survol de la géologie sur un petit nombre d'auteurs. Outre le manuel classique de Charles Lyell, dont une traduction française fait partie du Fonds ${ }^{74}$, et les travaux de William Logan sur les formations géologiques de l'Est du Canada ${ }^{75}$, les sources de Provancher sont surtout des ouvrages de vulgarisation, comme ceux de Dawson ${ }^{76}$; des textes anciens de polémique, ceux de William Conybeare ${ }^{77}$; des articles d'encyclopédie; des ouvrages d'apologétique et de théologie naturelle ${ }^{78}$.

Parce que sa discussion des hypothèses transformistes et son intérêt pour la géologie semblent subordonnés à la défense du dogme et aux exigences de l'apologétique, on peut croire que Provancher n'a pas senti la nécessité de bien pourvoir sa bibliothèque d'ouvrages sur ces matières, ni de la maintenir à jour. Quelques textes de référence, ou de vulgarisation, les traités classiques et les ouvrages d'apologétique de l'époque suffisent à son propos. Selon toute vraisemblance, la pauvreté du Fonds en livres plus savants sur la géologie et le transformisme correspond à celle de la bibliothèque originelle.

Il resterait à savoir de quels ouvrages de théologie naturelle, d'apologétique ou de philosophie, pouvait disposer Provancher, en plus de ceux cités dans le Naturaliste canadien. Sur cette section de la bibliothèque de Provancher, nous n'avons guère d'indica-

73 Nat. can. 10 (4) (1872): 309-310.

74 Éléments de géologie, traduit par J. Ginestou (Paris, Garnier, 6e édition, 1867).

75 Même si Provancher ne cite aucun des travaux de Logan en particulier, il s'agit probablement de l'un ou l'autre des nombreux Reports of Progress of the Geological Survey of Canada, documents annuels publiés depuis 1842 .

76 Op. cit., note 59

77 Conybeare s'était distingué, en Angleterre vers 1830, dans la controverse sur le Déluge mosaïque. Provancher ne cite que de nom Conybeare, dont il ne connaissait peut-être les travaux que de source secondaire, Nat. can., 6 (6) (1874) 168.

78 Sans autre précision, Provancher énumère Vossius, Mgr Meigan, le Père Caussette, l'abbé Lambert et jusqu'à Bède le Vénérable! 
tions. Il en va de même pour les ouvrages sur l'art, la littérature ou l'histoire que pouvait posséder l'éditeur du Naturaliste canadien. Cependant, nous savons que les auteurs canadiens, ou leurs éditeurs, lui faisaient souvent parvenir leurs livres. Ainsi, Provancher a-t-il eu l'occasion de rendre compte, dans les pages de sa revue, des opuscules du Dr Hubert LaRue sur l'agriculture, l'éducation des enfants ou l'histoire du Canada ${ }^{79}$; d'ouvrages de piété, tels l'Abrégé de la vie de Bernadette, Soeur Marie Bernard ${ }^{80}$; du Dictionnaire des familles canadiennes de Mgr Tanguay ${ }^{81}$; d'un roman d'Antoine Gérin-Lajoie, Jean Rivard économiste ${ }^{82}$; et même d'oeuvres de critique littéraire, comme celles de Tardivel $^{83}$ et de Charles Baillargé ${ }^{84}$.

Nous savons, enfin, que Provancher échangeait le Naturaliste contre la plupart des journaux québécois de l'époque. En 1873 et en 1877, il rédige des articles célèbres, qui n'ont d'ailleurs pas échappé aux historiens, dans lesquels il passe en revue la presse québécoise ${ }^{85}$.

\section{Conclusion}

S'il faut admettre que nous ne connaissons pas parfaitement la richesse de la bibliothèque de Provancher, nous pouvons néanmoins croire que le catalogue des ouvrages scientifiques, établi à partir du Fonds conservé à l'Université Laval, de la correspondance et de l'oeuvre du naturaliste, est à peu près complet. Cette bibliothèque suffisait-elle au travail de l'entomologiste et du malacologiste? Certains correspondants lui ont reproché, parfois méchamment, de ne pas maîtriser toutes les sources. Après avoir reçu le premier volume de la Petite Faune entomologique du Canada, qui traite des Coléoptères, Horn lui répond:

You would not try to make a watch in a blacksmith shop, than why try to describe new species and new genera without those books showing what has already been done? ${ }^{86}$

79 Petit manuel d'agriculture (1870), revue dans Nat. can., 12 (2) (1870), De la manière d'élever les jeunes enfants (1875), dans le Nat. can., (8) (1875), et Histoire du Canada (1875), dans le Nat. can., 7 (7) (1875).

80 Nat. can., 11 (11) (1879).

81 Nat. can., 4 (17) (1887): 63-64.

82 Nat. can., 6 (8) (1875).

83 Borrowed and Stolen Feathers (Québec, Imp. du Canadien, 1879).

84 Québec, passé, présent, futur (Québec, 1885, 8 pages).

85 «Nos journaux», Nat. can., 7 (5) (1873): 203-209, 8 (5) (1873): 335-341. «Notre presse», Nat. can., 5 (9) (1877): 129-156, 6: 177-195.

86 Lettre de Horn à Provancher, 12 juin 1877. APSC: Fonds Provancher. 
En 1885, c'est au tour d'un correspondant de Finlande, E. Bergroth, de lui signaler des ouvrages d'entomologie nord-américaine qui lui ont échappé ${ }^{87}$.

Parfois, c'est Provancher lui-même qui reconnaît les limites de sa bibliothèque. En 1891, il renonce à poursuivre la Petite Faune entomologique du Canada, pour laquelle il ne restait plus à étudier que les Lépidoptères, les Diptères et les Aptères.

C'est que pour écrire il faut avoir des auteurs, et que la bibliothèque du parlement est très pauvre en ouvrages scientifiques, elle est presque complètement dépourvue des ouvrages récents qui peuvent nous renseigner sur les productions naturelles de notre pays. Je possède plusieurs auteurs sur cet ordre d'insectes [Lépidoptères], mais le principal me manque; c'est l'histoire de ces insectes que publie actuellement M. Edwards aux États-Unis, avec de magnifiques gravures coloriées de chaque espèce, au prix de \$2.50 la livraison, une trentaine de livraisons ont déjà paru, et seront suivies probablement de 60 à 100 autres. Or si le gouvernement n'a pas le moyen d'acheter un tel ouvrage, comment veut-on que je me le procure moimême? ${ }^{88}$

Mais les critiques et les aveux de cette sorte ne sont pas fréquents et, en considérant l'ampleur de l'oeuvre taxonomique de Provancher dans le domaine de l'entomologie nord-américaine et de la malacologie,on peut déduire qu'il avait à sa disposition la plupart des ouvrages nécessaires.

La richesse de la bibliothèque scientifique de Provancher nous amène à faire quelques commentaires sur l'histoire du livre et des bibliothèques au Canada français. On peut remarquer que si plusieurs des ouvrages spécialisés de la bibliothèque Provancher, nécessaires à qui s'occupe de la systématique de certains groupes particuliers d'organismes, ne sont pas susceptibles d'apparaître au catalogue des bibliothèques publiques et des bibliothèques des maisons d'enseignement, ce n'est pas que la censure religieuse s'exercerait indistinctement à l'égard des livres de science, qu'il s'agisse d'ouvrages spécialisés ou de textes de polémique, mais que ces ouvrages sont de peu d'utilité au public de ces bibliothèques. L'enseignement des sciences à l'Université Laval et dans les collè-

87 Lettre de Bergroth à Provancher, 3 novembre 1885. APSC: Fonds Provancher.

88 Les Mollusques..., ii. 
ges classiques du Canada français est réduit à si peu de chose qu'il peut être exclusivement basé sur les manuels de Moyen, Brunet et Laflamme. Pour les bibliothèques publiques, les ouvrages encyclopédiques, les textes de vulgarisation scientifique et les traités classiques présentent davantage d'intérêt que les catalogues taxonomiques. Bref, la bibliothèque privée d'un naturaliste ne se constitue pas comme une bibliothèque générale publique, ni comme celle d'une maison d'enseignement.

Pour cette raison, on peut énoncer l'hypothèse que certaines des collections scientifiques les plus complètes, au moins pour un domaine, sinon les plus considérables, ont été constituées au siècle dernier, non pas par les bibliothèques publiques ou les collèges classiques, mais par des amateurs d'histoire naturelle, de physique, de chimie, d'astronomie, etc... Des amateurs de la bonne bourgeoisie canadienne-française, comme P.-J.-O. Chauveau, James M. Lemoine, le Dr LaRue, Charles Baillargé ou D.-N. Saint-Cyr ont pu, tout autant que Provancher, rassembler des bibliothèques

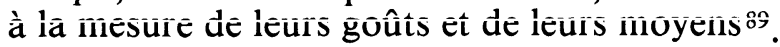

En plus des grandes bibliothèques scientifiques de la Literary and Historical Society of Quebec, de l'Université Laval et de la Natural History Society of Montreal, c'est du côté des bibliothèques privées qu'il faudra donc pousser les recherches sur la circulation et la diffusion des livres de science au Canada français au siècle dernier. Peut-être le rassemblement du Fonds Chauveau, présentement en cours à la Bibliothèque de l'Assemblée, nous fournira-t-il le second exemple d'une collection considérable d'ouvrages de science.

89 Chauveau s'intéressait à la géologie; Lemoine a publié quelques ouvrages de vulgarisation sur l'ornithologie; LaRue avait étudié la chimie à Louvain et à Paris avant de l'enseigner à l'Université Laval; Baillargé, membre fondateur de la Société royale du Canada, a compilé un gros ouvrage de géométrie et, enfin, Saint-Cyr a été pendant près de trente ans le Conservateur du Musée de l'Instruction publique. Il faut noter que cette liste d'amateurs n'est pas complète. Cf. Léon Lortie, "La trame scientifique de l'histoire du Canada», dans Pioneers of Canadian Science, 3-35. 